\title{
LA.7581
}

UC.20 J

Issued: March 1979

\section{An Approximate Model for Toroidal Force Balance in the High-Beta Stellarator} D. C. Barnes
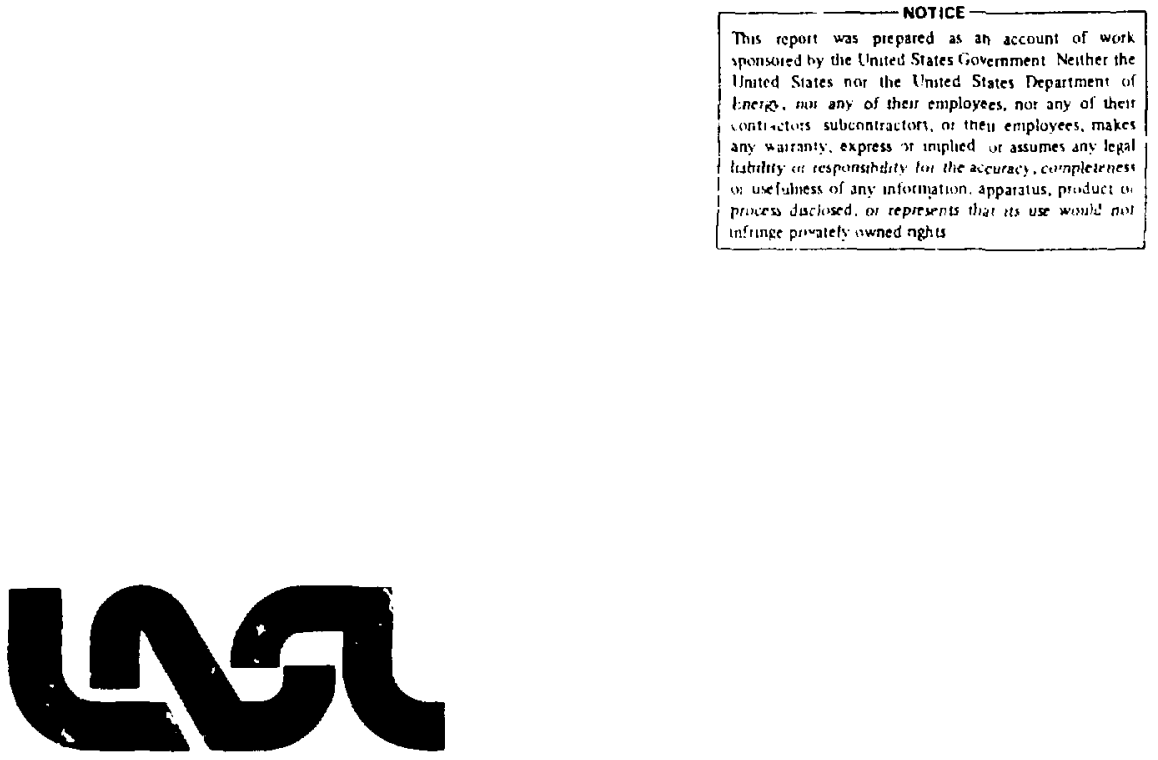
AN APPROXIMATE MODEL FOR TOROIDAL FORCE BALANCE

IN THE HIGH-BETA STELLARATOR

\author{
by \\ D. C. Barnes
}

\begin{abstract}
A simple model for estimating the body force acting on a diffuse plasma confined in a three-dimensional, high-beta stellarator geometry is given. The equilibrium is treated by an asymptotic expansion about a straight theta pinch with diffuse, circular cross section. The expansion parameter $\delta$ is the strength of the applied helical fields. This expansion leads to an inconsistent set of equations for the equilibrium in second order. Nevertheless, by averaging the equilibrium equations over the volume of the confined plasma, a unique condition for toroidal equilibrium is obtained. When the results are compared with the predictions of previous equilibrium theory, which is based on the sharp-boundary model, a large deviation is found. This correction is espectally large for $\ell=0,1$ systems at high beta and must be accounted for in any confinement experiment.
\end{abstract}

\title{
I. INTRODUCTICN
}

The Scyllac experiments ${ }^{1-4}$ at the Los Alamos Scienific Laboratory (LASL) and similar experiments (ISAR $T 1)^{5-7}$ at the Max-Planck Institute fuir Plasmaphysjk, Garching are toroldal plasma confinement systems of the high-beta stellarator type. In this geometry, a toroidal theta pinch is held In equllibrium by balancing the outward toroidal drift force against an fnward stellarator force. This later force is produced by the interaction of two helical fields with different symmetrles. The resulting configuration is inherently three-dimensional. 
To study this system theoretically, two approaches are useful. One technique represents the geometry on a three-dimensioral mesh and finds numerical solutions with a minimum of simplifying assumptions. This technique has been applied to the equilibrium equations ${ }^{8}$ as well as to the full t1me-dependent equations ${ }^{9}$ for magnetohydrodynamic flow. The second technique reduces the problem to one in fewer dimensions by an asymptotic expansion based on certain simplifying assumptions. The original theoretical basis for high-beta stellarator toroldal equilibria is of the second type.10,11 The asymptotic expansion is based on the constant plasma pressure, skin-current model. For this profile, under the additional assumptions of small helical distortions or long helical wavelength (or both), the equilibrium conditions reduce to an algebraic set of conditions matching two vacium fields at the plasma surfice.

The extension of such an expansion to the more realistlc case in which the plasma profile is diffuse introduces serious complications. Davidson, Frefdterg and Berge, 12 and Weitzner, ${ }^{13}$ with two independent expansions, sought equilibria that are perturbations of a straight theta pinch with diffuse, circular crcss section. The restriction to variation of the lowest order proflie only in the radial direction leads to a very small class of solutions, wit:L s:o not particularly Interesting physically. More recently, Barnes et a.. 14 have proposed an expansion about a straight configuration with two-dimensional variation In the lowest order profile. Reasonable solutions probably exist, but the increase in complexity makes their computation more difficult.

The work presented here has a more modest goal than computing these equilibria in detail. Nauely, if we assume that such equilibria exist, how may the gross features of the resulting co.figurations be estimated? In particular, what fields must be applied externally for an equilibrium to be attalned?

A technique for obtaining such an estimate for the required flelds is presented here. Several simplifylng assumptions are made. The toroldal case is assumed co be a perturbation of a linear theta pinch with circular cross section but with an arbitrary profile. This assumption generally resuit.s in an inconsistent set of equations for the equilibrium in second orde... However, if second-order force balance is required only in a volume averaged sense, a unique field product for equilibrium is deterinined. 
Although some discussion is given, the effects of the averaged-out Imbalanced local forces are largely ignored. The averaged results indicate only what should be expected from consistent equilibrium solutions. In a recent comparison with three-dimensional numerical results, 15 the estimate presented here seems to predict the equilibrium parameters remarkably well.

The remainder of the report is djvided into three sections. In Sec. II, the molel for toroidal force balance is derived. Section III presents the results of the solution of the force-balance condition for sharp boundary and diffuse profiles. In Sec. IV, conclusions relevant to experimental observations and further theoretjcal work are glven.

\section{ASYMPTOTIC MODEL}

A toroldal theta pinch with a circular cross section, a very large aspect ratio, and small. perturbing helical fields is described by the distorted cylindrical coordinates, $r, \theta$, and $z$ (Fig. 1 ). The axlal coordinate $z$ is the distance measured along a circle of radius $R_{0}$, which is the minor axis of the torus. In any plane normal to this circle, $r$ and $\theta$ are the usual planar polar coordinates with origin at the minor axis and the zero of $\theta$ along the direction away frow the major axis of the torus.

The ordering assumed is similar to the "old scyllac" ordering. 10,11 We take

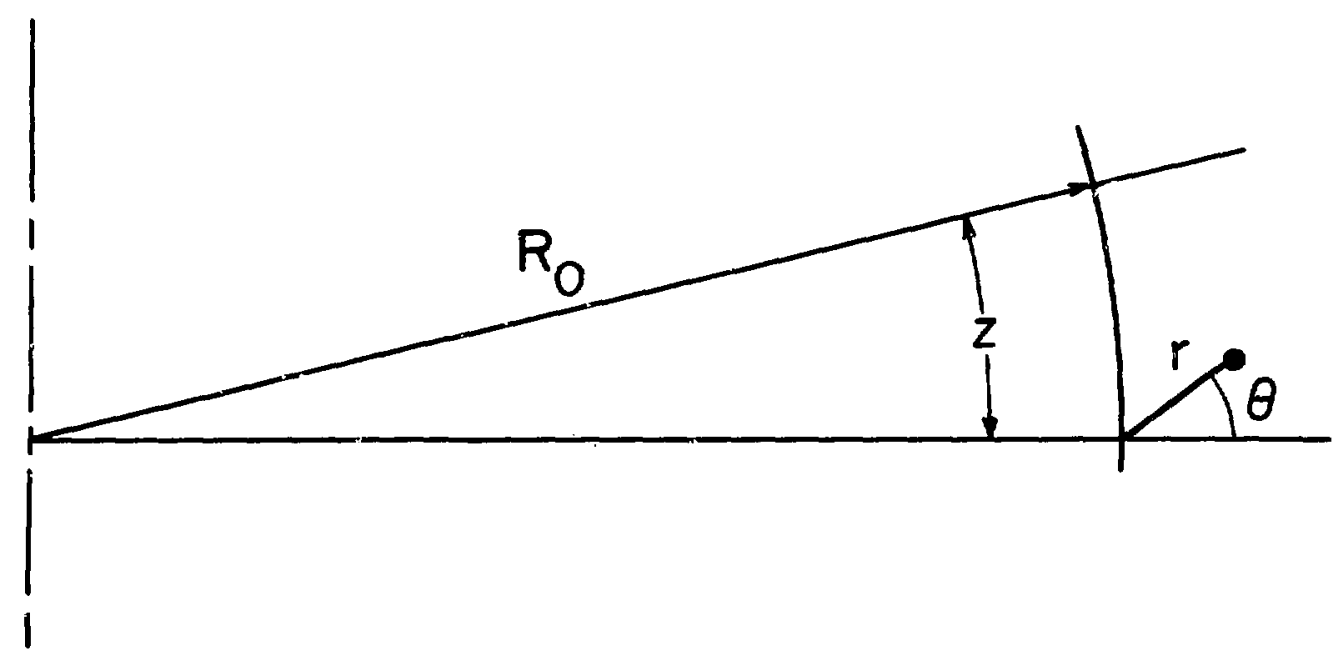

Fig. 1

Pseudo-toroidal coordinates for describing confinement geometry. 


$$
\frac{a}{R_{0}}=O\left(S^{2}\right) ;\left|B_{l}\right|=O(\delta) ; \& \ll 1
$$

where a 18 a characteristic minor radius of the plasma and ${\underset{\sim}{\ell}}_{\ell} 18$ any of the applied helical flelds.

For an arbitrary cross section, the magnetic field is expanded to second order as

$$
\underset{\sim}{B}=B(r, \theta) \hat{z}+\sum_{\ell} \underset{\sim}{B_{\ell}}+{\underset{\sim}{B}}^{(2)}+\ldots,
$$

where $B(r, \theta)$ is the zeroth-order theta pinch fleld. The ${\underset{\sim}{\ell}}_{\ell}$ are first-order helical flelds depending on $\theta$ and $z$ only through the heilcal varlable $\Phi_{\ell}=\ell \theta-h z$, where $\ell 1 s$ an integer and $h$ is the helical wave number, $h=2 \pi / L$, where $L 1 s$ the helical periodic length. The third term above represents flelds arising from the weak toroldal curvature and any other second-order flelds.

The equation for a magnetostatic equilibrium,

$$
\nabla P=(\nabla \times \underset{\sim}{B}) \times \underset{\sim}{B},
$$

where $P$ 1s the plasma pressure, may be expanded order by order. The lowest order terms relate the gradient of the zeroth order pressure, $P(0)$, to $B$. These conditions may be integrated to give

$$
P^{(0)}+\frac{1}{2} B^{2}=\text { constant, }
$$

just as for a linear theta pinch.

To first order in $\delta$, the various hellcal flelds couple unless we assume that the zeroth-order field $B(r, \theta)$ depends only on $r$. With this assumption, the ${\underset{\sim}{B}}_{\ell}$ may be considered separately. For this, the conds.tion $\nabla^{*} \underline{\sim}_{\ell}=0$ is integrated by Introducing the flux function $\psi_{\ell}(r)$ according to 


$$
B_{\ell \mathbf{r}}=-\frac{\Psi_{l}}{\mathbf{r}} \sin \phi_{\ell}
$$

and

$$
\frac{\ell}{r} B_{\ell \theta}-h E_{\ell z}=\frac{\psi_{l}}{r} \cos \phi_{\ell} .
$$

Differentiation with respect to $r$ is denoted by a prime here and below.

From Eq. (2), we obtain three component equations involving $\underline{B}_{\ell}$ and $P_{\ell}=P_{\ell}\left(r, \phi_{\ell}\right)$, the first order perturbed pressure assoctated with the $\ell$ th helical fleld. Because of the ordering, toroldal curvature does not enter in first order. Thus, the above equations are the same as those obtained from a small. amplitude expansion of a straight helical equilibrium about a linear theta pinch.

After some algebraic manipulation of the first-order equations, $P_{\ell}$ is eliminated. Using Eq. (4), the following equation for $\psi_{l}$ is obtained.

$$
\left(\frac{x \psi_{\ell}^{\prime}}{D_{\ell}}\right)^{\prime}-\left[\frac{\left(r B^{\prime}\right)^{\prime}}{B}+\frac{1}{r}\right] \Psi_{\ell}=0,
$$

where $D_{\ell}$ is given by

$$
D_{\ell}=\ell^{2}+(h r)^{2}
$$

Equation (5) has been previously derived by Berge and Freidberg ${ }^{16}$ for a stralght helical system.

Boundary conditions for $\psi_{\ell}$ are obtained by specifying the small distortions of an almost cylindrical conducting wall at $r=b$. The boundary condition at this conductor is $\underset{\sim}{\mathrm{B}} \mathrm{Z}=0$, where $\mathrm{n}$ is the unit normal to the wall. To ffrst order in $\delta$, this gives

$$
\psi_{\ell}(\mathrm{b})=\mathrm{hb}^{2} \mathrm{~B}(\mathrm{~b}) \Delta_{\ell,}
$$


where the radius of the slightly distorted wall is given by

$$
\mathbf{r}=b\left(1-\sum_{\ell} \Delta_{\ell} \cos \phi_{\ell}\right)
$$

Equation (7) provides one boundary condition for the second-order differential equation (5). A second boundary condition resilts from the requirement that $\psi_{\ell}$ be finite at $r=0$. To examine this, consider Eq. (5) near $r=U$. In this region, the differential equation simplifies since $B^{*}+0$. From Eq. (5), the resulting asyptotic equation is

$$
\left(r \psi_{\ell}^{\prime} / D_{\ell}\right)^{\prime}-\psi_{\ell} / \mathrm{r} \sim 0
$$

Solutions to Eq. (9) may be givnn in terms of the modified Bessel functions $I_{\ell}$ and $K_{\ell}$ as

$$
\psi_{l} \sim r\left[A_{1} I_{l}^{\prime}(h r)+A_{2} K_{l}^{\prime}(h r)\right] .
$$

Regularity at $r=0$ now has a clear meaning. In order for $\psi_{\ell}$ to remain finite as $r \rightarrow 0, A_{2}$ must vanish. Thus

$$
\psi_{\ell} \sim A_{1} r I_{\ell}^{\prime}(h r)
$$

Since $r$ is swall, the Bessel function above may be expanded to give the second boundary condition for $\psi_{\ell}$,

$$
r \psi_{\ell}^{\prime}=\ell \psi_{\ell}, \ell \neq 0
$$

and

$$
\text { as } \mathbf{r} \rightarrow 0
$$

$$
r \psi_{0}^{\prime}=2 \psi_{0}
$$


Equation (9) also describes the region $r / a \gg 1$, where again, $B^{\circ}+0$. It is convenient to write the asyiaptotic solution for this region as

$$
\begin{aligned}
& \psi_{\ell} \sim \frac{\mathrm{B}_{0} \varepsilon \mathrm{r}}{\ell}\left[c_{\ell} \frac{2^{\ell} \ell !}{\varepsilon^{\ell}} I_{\ell}-d_{\ell} \frac{\varepsilon^{\ell}}{2^{\ell-1}(\ell-1) !} \mathrm{K}_{\ell}^{\dot{C}}\right], \ell \neq 0 \\
& \psi_{0} \sim B_{0} r\left[c_{0} \frac{I_{0}}{\varepsilon}-d_{0} \frac{\varepsilon \mathrm{K}_{0}}{2}\right]
\end{aligned}
$$

where the Bessel functions are evaluated at $\mathrm{hr}$, where $\varepsilon=$ ha $1 \mathrm{~s}$ the dimensionless helical wave number, and where $B_{0}$ is the limiting value of $B(r)$ as $r+\infty$. The normalization 1 s chosen so that the above forms become particularly simple when $E \ll 1$,

$$
\psi_{\ell} \sim \mathrm{aB}_{0}\left[c_{\lambda}(r / a)^{\ell}+d_{\ell}(r / a)^{-\ell}\right], \ell \neq 0
$$

and

$$
\psi_{0} \sim \mathrm{aB}_{0}\left[\frac{1}{2} c_{0}(\mathrm{r} / \mathrm{a})^{2}+\frac{1}{2} d_{0}\right]
$$

The significance of the constants $c_{\ell}$ and $d_{\ell}$ which characterize the field outside the main plasma column will become apparent in the following discussion of second-order forces.

All Information about toroldal equllibrium is contained in the second order, that 18, $O\left(\delta^{2}\right)$ terms, which are considered now. These terms result from the Interaction of nelghboring helical flelds, pairs $\underline{\sim}_{\ell}, \underline{a}_{\ell+1}$, and from the efrect of toroidal curvature on the lowest order $z$ field. As pointed out by Davidson and Freidberg, 12 the terms causing difficulties are those that are Independent of $z$. If the $O\left(\delta^{2}\right)$ terms from Eq. (i) are averaged over $z$ the following equations result. 


$$
\begin{aligned}
& F_{11 r}=\frac{\partial P(2)}{\partial r}+B^{\prime} B_{z}^{(2)}+B \frac{\partial B_{z}^{(2)}}{\partial r}+\frac{B}{R} \cos \theta, \\
& F_{11 \theta}=\frac{1}{r} \frac{\partial P(2)}{\partial \theta}+\frac{1}{r} B \frac{\partial B_{z}^{(2)}}{\partial \theta}-\frac{B}{R} \sin \theta,
\end{aligned}
$$

and

$$
F_{11 z}=-B^{2} B_{r}^{(2)}
$$

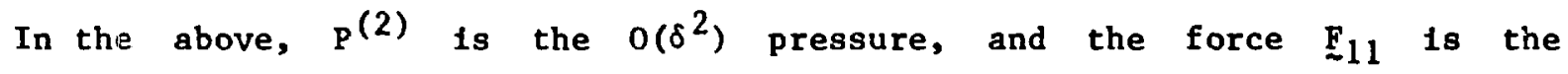
contribution from the first-order field alone, averaged over one helical period

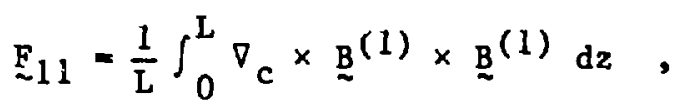

where $\mathrm{g}^{(1)}$ is the total first-order field and the curl operator does not Include toroldal curvature; it is the usual cylindrical operator.

From the first two expressions of Eq. (13) follows

$$
\frac{\partial F_{11 r}}{\partial \theta}-\frac{\partial\left(r F_{11 \theta}\right)}{\partial r}=\frac{r B B^{\circ}}{R} \sin \theta .
$$

The difficulty with the second-order equations may be seen from Eq. (14). The first-order fleld for each $\ell$ is determined everywhere by the solution of Eq. (5), once the single constant $\Delta_{\ell}$ is given. On the other hand, Eq. (14) gives another condition which $\psi_{\ell}$ must satisfy at each value of $r$. This is not possible unless the lowest order profile $B(r)$ is chosen in a special way. This constraint prevents the specification of physically interesting profiles. A simple way to avold the constraint of: Eq. (14) and salvage some information from the expansion is to weaken the requirement for equiltbrium in second order. Suppose Eq. (2) is integrated over the volume of the conftined plasma, and only this total force must vanish. Then any local inconsistencies are avolded and a unique condition for an averaged equflibrium results. 
Since the reader might well be concerned with the significance of results obtained by such a gross simplification, a short digression is appropriate. Consider the equilibrium being studied as the final state of a time-dependent, toroldal, magnetohydrodynamic system with applied flelds of the form given by Eq. (1). Terms of various orders in the equilibrium equations correspond to various time scales in the time-dependent problem.

The $O(1)$ and $O(\delta)$ terms lead to rapid $z$-independent and helical motions, respectively. In a short time, these motions will be dissipated in any real plasma, so that the motion at later times is determined by higher order forces. With the lower order fields satisfying Eq. (2), a quasi-static, z-Independent motion force by the second-order terms changes the plasma profile slowly until the equilibrium condition is satisfled everywhere. Because of conservation of magnetic flux and pressure balance [Eq. (3)], this long time motion must be incompressible. Thus, the average distributions of magnetic and internal energy are unaffected.

Force balance in the volume averaged sense is characterized by the gross features of the $O(\delta)$ response. If this response depends relatively little on the details of the profile and morc directly on the average distribution of fleld and pressure, the assumption of circular cross section in lowest order is justified.

In fact, for small values of $\ell$, the helical iflid penetrates the entire plasma volume, so that the flasma response should not be sensitive to the detalls of the profile. For at least one case, the plasma response can be shown to depend only on the average features of the profile. This is the case of a bumpy $(l=0)$ distortion of a noncircular theta pisch when $\varepsilon \ll 1$. A similar insensitivity might be expected for small but nonzero values of $\ell$. Thus, the estimates obtalned by volume averaging should apply to those systems which are most interesting experimentally, that 1s, those with applied $\ell=0,1$ and $\ell=1,2$ helical flelds. Since any further fustification of the model would carry the discussion too far from the main content of this report, we return now to the derivation of the force balance condition.

If the magnetohydrodynamic force density is integrated over a control volume conialning one hellcal perlod of the plasma column, the resulting force per unit length is given by the surface integral of the total stress (materlal plus Maxwe11) as 


$$
L \underset{\sim}{F}=\int_{S}-\left(P+\frac{1}{2}|\underline{\sim}|^{2}\right) d \underset{\sim}{A}+\underset{\sim}{B}\left(B^{\cdot} \cdot d \underset{\sim}{A}\right),
$$

where $\underset{\sim}{F}$ is the total force per unit length, and $S$ is the surface of the control volume with outward oriented area element dA. The control volume is chosen to be the smooth toroidal sector, $0<z<L, r<r_{c}$, where $r_{c} / a>1$. The surface $S$ consists of two parts, the curved outer surface $T$ and the two ends E. Contributions to the second-order force from these two portions resuit from different terms in the stress. On the surface $T$, the pressure $P$ vanishes because the surfaco is chosen far from the plasma. We need to retain all second-order terms arising from the magnetic field $\underset{\sim}{\mathrm{B}}$. On the ends $\mathrm{E}$, both $P$ and $\underset{\sim}{B}$ contribute to $\underset{\sim}{F}$. However, because of periodicity of all guantities except the area element $\mathrm{dA}_{\sim}$, the contributions from the two ends cancel thru $O(\delta)$. Hence, on the ends, only the lowest order terms in $P$ and $B$ are needed to compute the second-order force $\mathrm{F}$.

It is convenient to combine terms from the toroldai fleld alone and those from the helical fields. Only the radially outward $(\theta=0$ direction) component of the force is nontrivial. The contribution from the toroldal field produces a radially outward force per unit length,

$$
F_{R}=\frac{1}{R_{0}}\left\{2 \pi \int_{0}^{r_{c}}\left(P^{(0)}-\frac{1}{2} B^{2}\right) r d r+\frac{\pi B_{0}^{2} r_{c}^{2}}{2}\right\},
$$

where the first term is the contribution from $E$ and the second arises from the variation in area element and fleld strength over $T$. In dertving the second term, the condition that no current flow through the outer surface $T$ is used. This requires that a second-order field

$$
{\underset{\sim}{B}}^{(2)}=-\frac{r \cos \theta}{R_{0}} B_{0} \hat{z} \text {, }
$$

be present to account for the toroldal curvature. 
If pressure balance (Eq. (3)) is used in Eq. (16), the final form for the toroldal drift force per unit length is obtained

$$
F_{R}=\frac{2 \pi B_{0}^{2}}{R_{0}} \int_{0}^{r_{r}} \beta(r) r d r
$$

where the global $\beta$ is defined by

$$
B(r)=2 P^{(0)}(r) / B_{0}^{2}
$$

The expression for $F_{R}$ in Eq. (18) was first given by van der Laan. 17

Neighboring pairs of helical fields give a contribution to $\mathrm{F}$ from $\mathrm{T}$. For a pair $\ell, \ell+1$, the radially outward force per unit length is obtained from Eqs. (15) and (4). After some algebra

$$
\begin{aligned}
& F_{\ell, \ell+1}=-\frac{\pi B_{0}^{2}}{2 r_{c}}\left\{\psi_{\ell} \psi_{\ell+1}+\frac{(\ell+1) r_{c} \psi_{\ell} \psi_{\ell+1}}{D_{\ell+1}}\right. \\
& -\frac{\ell \mathbf{r}_{c} \psi_{\ell}^{\prime} \dot{U}_{\ell+1}^{\prime}}{\mathrm{D}_{\ell}}-\frac{\mathrm{r}_{c}^{2}\left[\ell(\ell+1)+\left(\mathrm{hr} \mathbf{r}_{c}\right)^{2}\right] \psi_{\ell}^{\prime} \psi_{\ell+1}^{\prime}}{\mathrm{D}_{\ell} \mathrm{D}_{\ell+1}},
\end{aligned}
$$

where all quantities are evaluated at $r=r_{c}$ *

The significance of the asymptotic form for $\psi_{\ell}$ given by Eq. (11) becomes clear if these expressions are substituted Into Eq. (20)

$$
F_{\ell, \ell+1}=-2 \pi \mathrm{aB}_{0}^{2}\left[\frac{d_{\ell}}{c_{\ell}}+\frac{\varepsilon^{2}}{4 \ell(\ell+1)} \frac{d_{\ell+1}}{c_{\ell+1}}\right] c_{\ell} c_{\ell+1}, \ell \neq 0
$$

and 


$$
F_{0,1}=-\frac{\pi a B_{0}^{2}}{2}\left[\frac{d_{0}}{c_{0}}+\frac{d_{1}}{c_{1}}\right] c_{0} c_{1} .
$$

The constants $c_{\ell}$ and $d_{\ell}$ have the significance of the applied hellcal fleld and the diamagnetic helical field resulting from the plasma response, respectively.

The toroldal force balance condition ls obtained by combining Eqs. (18) and (21). Since the total radially outward force must vanish, we obtain

$$
\begin{aligned}
\frac{1}{R_{0}} \int_{0}^{r_{c}} B(r) r d r & =a\left[\frac{d_{\ell}}{c_{\ell}}+\frac{\varepsilon^{2}}{4 \ell(\ell+1)} \frac{d_{\ell+1}}{c_{\ell+1}}\right] c_{\ell} c_{\ell+1}, \ell \neq 0 \\
& =\frac{a}{4}\left[\frac{d_{0}}{c_{0}}+\frac{d_{1}}{c_{1}}\right] c_{0} c_{1}, \ell=0 .
\end{aligned}
$$

From the ratios $d_{\ell} / c_{\ell}, d_{\ell+1} / c_{\ell+1}$ and the volume integrated $\beta$, the hellcal fleld product, $c_{\ell} c_{\ell+1}$, required for coroldal force balance is determined from Eq. (22). A final step required to relate this fleld product to that required In an exisimental situation is to include the effect of the finite distance of the outer conducting wall from the plasma. It 18 convenient to represent the helical fields required experimentally by giving their value near the axis In vacuum, because the vacuum field produced by any given coll configuration may be calculated more or less directly.

In order that the $\ell$ th helical field component has strength $c_{\ell}$ when plasma is present, the $\Delta_{\ell}$ of the outer wall is determined by the outer boundary condition using Eqs. $(7)$ and (11). In vacuim, the fleld strength will be $\dot{c}_{\ell}$, again determined from the outer boundary condition but with $\mathbf{d}_{\ell}=0$ in Eq. (11). A comparison of the two cases gives the correction factor $f_{\ell}>1$ as 


$$
\begin{aligned}
f_{\ell}=\dot{c_{l}} / c_{\ell} & =1-\frac{2}{\ell !(\ell-1) !}(\varepsilon / 2)^{2 \ell} \frac{K_{\ell}^{\prime}}{I_{\ell}^{\prime}} \frac{d_{\ell}}{c_{\ell}}, \ell+0 \\
& =1-\frac{\varepsilon^{2}}{2} \frac{K_{0}^{\prime}}{I_{0}^{\prime}} \frac{d_{0}}{c_{0}}, \ell=0,
\end{aligned}
$$

where the Bessel functions are evaluated at hb.

In the next section, the force balance condition of Eq. (22) 1s considered for several spectfic profiles, and some comparative results are obtained. Before leaving the general discussion, however, some scailng properties of the force balance model are 1llustrated here.

For simplicity, the case $\varepsilon \ll 1$ 1s assumed. Consider first the normalization of the constants $c_{\ell}$, which represent the applied helical fields. If $\ell \neq 0$, it follows from Eqs. (12) and (4) that

$$
c_{\ell}=\frac{B_{\ell} r}{B_{0}}(r=a)
$$

is the usual normalization shosen for the applied helical fieids. (In previous literature, $c_{q}$ is usually denoted by $B_{\ell=q} / B_{0}$ for each integer $q>0$. ) If $\ell=0$, it follows in a similar manner that

$$
c_{0}=\varepsilon \frac{\delta B_{z}}{B_{0}}(r=0),
$$

which is $\varepsilon$ times the usuai normalized $\ell=0$ field $\left(c_{0}=\varepsilon B_{\ell=0} / B_{0}\right.$, where $B_{\ell=0} / B_{0}$ is the relative variation of the axial field on the axis). 
Now consider how the force balance condition scales with the plasma radius a. Since a is only a characteristic neasure of the width of the plasma profile, its value is somewhat arbitrary. On the other hand, a appears explicitly in Eq. (22). Thus, it is reasonable to ask how the force balance scales if the profile $\beta(r)$ and the applied helical flelds remains fixed as the value of a varies. It is apparent that the left hand side of Eq. (22) remains constant as a is varied. Since the helical fleld is fixed as a function of $r$, the flux $\Psi_{\ell}$ is fixed also, and 1 f follows from Eq. (12) that $c_{\ell} \propto a^{\ell-1}$ anu $d_{\ell} \propto a^{-\ell-1}$ for $\ell \neq 0$, and that $c_{0} \propto a$, and $d_{0} \propto a^{-1}$. Using these relations in Eq. (22) It is seen that the right-hand side is also independent of a. Thus, force balance, as $i t$ must, depends only on the physical situation and not on the cholce of the plisma radius a.

A more interesting situation arises if we assume that the entire profile Is scaled in radius, $\beta^{(\alpha)}(r)=\beta(r / \alpha)$, while the applied helical fields are held f:xed. For $\ell \neq 0$, Eq. (5) is approximately

$$
\left[r \psi_{\ell}{ }^{(\alpha)^{\prime}}\right]^{\prime}-\left[\frac{\left[\mathrm{rB}(\alpha)^{\prime}\right]^{\prime}}{{ }_{B}(\alpha)}+\frac{\ell^{2}}{r}-\right\} \psi_{\ell}^{(\alpha)}=0,
$$

since $\varepsilon \ll 1$. It is easy to verify that $\psi_{\ell}{ }^{(\alpha)}(r)=R \psi_{l}(\alpha r)$ is the solution of Eq. (26) for some constant $K$. The constant $K$ may be included in the definition of a by our previous considerations. Referring again to Eq. (12), the relative response $\left(d_{\ell} / c_{\ell}\right)^{(\alpha)}$ scales as $\alpha^{2 \ell}$.

Since the left hand side of Eq. (22) is a volume integral, this term scales as $\alpha^{2}$. Hence, for an $\ell=1,2$ system, the force balance is independent of the actual width of the profile and depends only on the shape. For any other $\ell>1$ pair, force balance exists for only a particular profile with a specifled shape and width. Although the $\ell=0,1$ case has not been considereil, It can be shown by similar arguements that force balance is also independent of the plasma width for this case.

\section{FOHCE BALANCE RESULTS}

In this section, the force balance condition of Eq. (22) is studied for various profiles, $B(r)$. The constants $c_{\ell}$ and $d_{\ell}$ that enter the force balance are determined by numerically integrating the differential Eq. (5) from $r=n$ 
where $n / a \ll 1$. Regularity gives the initial conditions of En. (10) from whtch the integration is started. At some final radius $r_{f}$, with $r_{f} / a \gg 1$, the solution obtained is compared with the asymptotic form of Eq. (11) and the ratio $\mathrm{d}_{\ell} / \mathrm{c}_{\ell}$ is obtained. This ratio is independent of the outer boundary condition of Eq. (7) since all solutions are proportional as $\dot{\Delta}_{\ell}$ is varied.

From the ratios $d_{\ell} / c_{\ell}, d_{\ell+1} / c_{\ell+1}$ and a numerical integration of $\beta$ over the volume of the plasma, the vacuum helical field product, $c_{\ell} \dot{c}_{\ell+1}$, required for toroldal force balance is determined from Eq. (22) with the wall corrections given by Eq. (23).

The force balance condition is first investigated for the case of an almost sharp-boundary profile. In this case, a profile, $\beta(r)$ is chosen so that $B$ is nearly constant for $r<a$ and nearly zero for $r>a$, with a smooth but steep transition near $r=a$. The actual profile used is given by

$$
B(r)=B(0)\left\{1+\exp \left[\frac{1}{\Delta}\left(\frac{r}{a}-\frac{a}{r}\right)\right]\right\}^{-1},
$$

where $\Delta$ measures the relative width of the transition sheath at $r=a$. In Fig. 2, this profile is shown for several values of $\Delta$.

For a sharp-boundary profile, the averaged force balance results coincide with the results of a consistent asymptotic expansion. The inconsistency discussed in the orevious section does not occur because eaullibrium requires

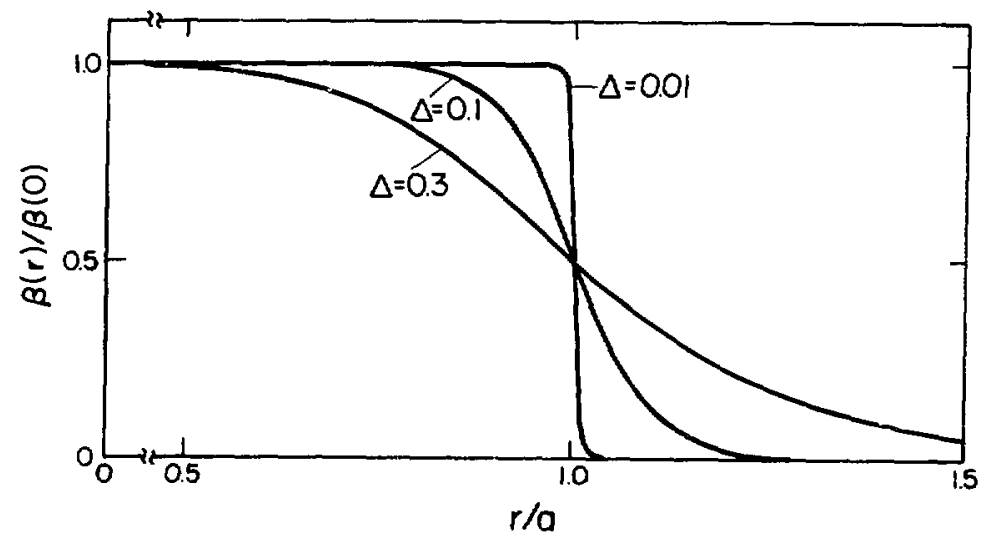

Fig. 2

Sharp profile used for several values of the sheath width parameter, $\Delta$. 
that a condition be satisfled at only one flux surface. Thus, force balance results obtained here should converge to those predicted by previous sharp-boundary theory as $\Delta \rightarrow 0$.

In Table I, the results of calculations for $\ell=0,1$ oystems with four values of $\Delta$ are shown along with those predicted by sharp-boundary theory. The quantity tabulated is the required helical field product for force balance, $\left(R_{0} / a\right) c_{0} c_{1}=h R_{0}\left(B_{\ell=0} / B_{0}\right)\left(B_{\ell=1} / B_{0}\right)$, versus the plasma $B$ on axis, $B(0)$. To simplify comparison, a very large ratio wall to plasma radius $(20: 1)$ and a small $\varepsilon(0.01)$ were chosen.

The numerical results converge to the sharp-boundary predictionis and the convergence is nonuniform in $B$. At high beta the effect of finite sheath width is much greater than at lower $B$. Similar results are ohtained for $\ell=1,2$ systems. Table II glves the required fleld product, $\left(R_{0} / a\right) c_{1} c_{2}=\left(R_{0} / a\right)\left(B_{\ell=1} / B_{0}\right)\left(B_{\ell=2} / B_{0}\right)$, versus plasma $B$ as obtained from sharp-boundary theory and from the averaged force balance model with a very thin sheath.

The force balance results for diffuse profiles indicate some striking differences from the sharp-boundary results. Two diffuse profiles were used. The first is the rigid rotor profile,

$$
B(r)=\operatorname{sech}^{2}\left[\left(\frac{r}{a}\right)^{2}+\Gamma\right],
$$

TABLE I

FIELD PRODUCT, $Q$, FOR $\ell=0,1$ EQUILIBRIUM

$\underline{B(0)} Q($ Theory $) Q(\Delta=.001) Q(\Delta=.01) \quad Q(\Delta=.1) \quad Q(\Delta=.3)$

$\begin{array}{llllll}0.01 & 1.322 & 1.322 & 1.323 & 1.323 & 1.354 \\ 0.1 & 1.221 & 1.222 & 1.223 & 1.233 & 1.279 \\ 0.3 & 0.993 & 0.993 & 0.996 & 1.022 & 1.102 \\ 0.5 & 0.752 & 0.753 & 0.756 & 0.796 & 0.903 \\ 0.7 & 0.490 & 0.492 & 0.495 & 0.543 & 0.669 \\ 0.9 & 0.188 & 0.189 & 0.192 & 0.231 & 0.344 \\ 0.99 & 0.025 & 0.025 & 0.026 & 0.036 & 0.077\end{array}$


TABLE II

FIELD PRODUCT, Q, FOR $\ell=1,2$ EQUILIBRIUM

$\begin{array}{lcc}\underline{B(0)} & \underline{Q(\text { Theory })} & \underline{Q(\Delta=.001)} \\ 0.01 & 0.995 & 0.995 \\ 0.1 & 0.950 & 0.951 \\ 0.3 & 0.850 & 0.851 \\ 0.5 & 0.750 & 0.751 \\ 0.7 & 0.650 & 0.652 \\ 0.9 & 0.550 & 0.552 \\ 0.99 & 0.505 & 0.506\end{array}$

where $\Gamma$ is determined $\operatorname{from} \beta(0)=\operatorname{sech}^{2} \Gamma$. The second is a Gaussian profile

$$
B(r)=B(0) \exp \left[-(x / a)^{2}\right] .
$$

E1ther of these profiles can be used to fit experimentally observed profiles. Table III gives the field product for $l=0,1$ diffuse equilibria for the two profiles as a function of $\beta(0)$. The results are normalized to the sharp-boundary fleld product given earlier. Thus, the numbers give the factor by which the helical field product must be increased from the sharp-boundary prediction to produce a diffuse equilibrium. In F1g. 3 , these correction

TABLE III

FIELD PRODUCT FOR DIFFUSE $\ell=0,1$ EQUILIBRIA

$\underline{B(0)}$

0.01

0.1

0.3

0.5

0.7

0.9

0.99
RIGID $\underline{\text { ROTOR }}$

1.005

1.046

1.160

1.334

1.659

2.757

7.568

\section{GAUSSIAN}

1.005

1.044

1.156

1.336

1.701

3.162

14.022 


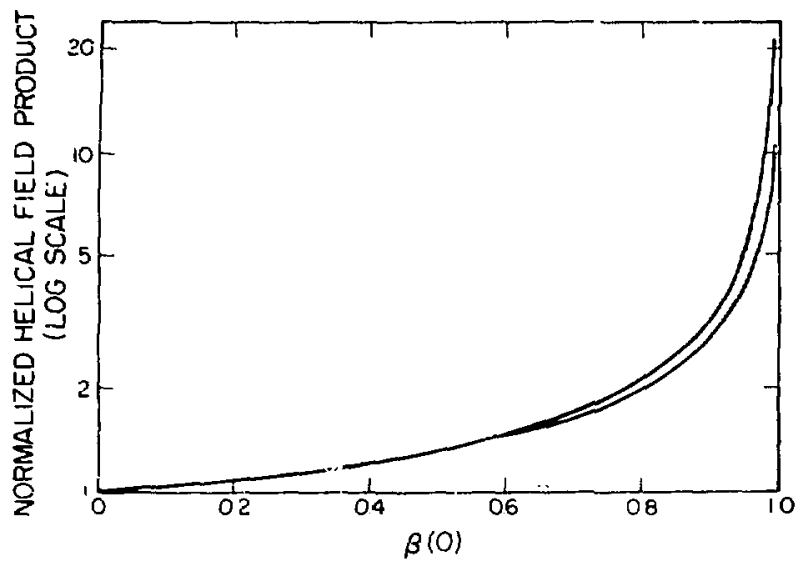

Fig. 3. Correction factor for helical field product required for two diffuse profile cases compared to sharp-boundary results.

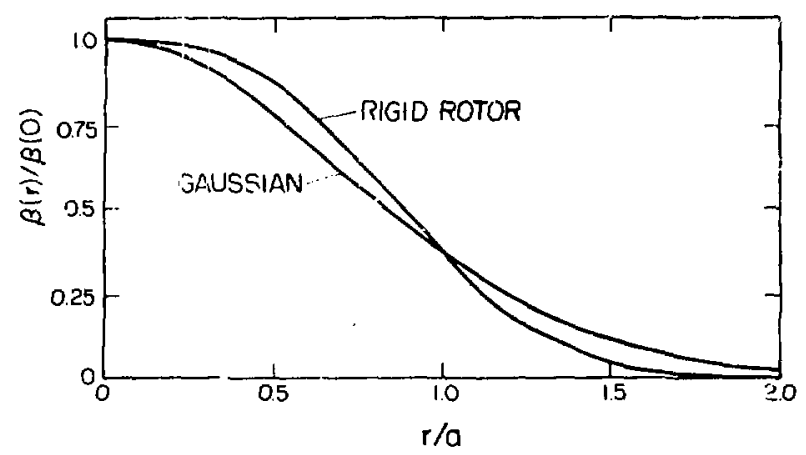

Fig. 4. Comparison of the detailed structure of rigid rotor and Gaussian profiles for a $\beta$ on axis of 0.95 .

factors are plotted versus the plasma $\beta$ on axis. Note that a logarithmic icale has been used to represent the extreme range of the correction factor.

As can be seen from the figure, force balance is not terribly sensitive to the details of the diffuse profile. The correction factor from a sharp-boundary plasma is much greater than the ratio of the Gaussian to the rigid rotor results. The deviation at higher $\beta$ 's of the results for the two diffuse profiles studied may be understood if thetr shapes are compared in detail. Figure 4 shows the two profiles for a $\beta=0.95$ on axis. The characteristic radius in each case is adjusted so that $\beta(1)=\beta(0) / e$. The rigid rotor profile is sharper than the Gaussian profile. This is consistent with a smaller helical field product required for the rigid rotor force balance.

Experimentally, the most interesting range of $\beta$ is roughly $0.65<\beta<0.95$. In this range a large increase $(1.5$ to 3 times the sharp-boundary value) in the helical field product is necessary to produce toroidal force balance for a diffuse plasma. This large factor makes an experimental test of this theory quite easy. In fact, an experiment using the helical flelds approprlate for a sharp-boundary plasma would not come close to producing a diffuse toroldal equilibrium. These predictions are consistent with experimental observations. ${ }^{4-6}$ A comparison of these results with experimental data is given in Ref. 15. 
In contrast to the $\ell=0,1$ case, the diffuse $\ell=1,2$ cases agree much more closely with sharp-boundary theory. Table IV gives the correction factors for diffuse $\ell=1,2$ cases as a function of $\beta(0)$. Even at the highest $P$.'s the correction to the sharp-boundary value is only 20-30\%. Although this deviation is st1ll measurable experimentally, it is much less important thai the $\ell=0,1$ correction factor discussed above.

\section{CONCLUSIONS}

The asymptotic model developed gives a prediction for the externally applied helical fields required for diffuse, toroldal, high-beta stellarator equilibria. A large devlation from the predictions of the sharp boundary, skin-current model, 10,11 which formed the original theoretical basis for this configuration, is found. This applies especially to $\ell=0,1$ systems at high beta.

Although the averaged results are not coppletely self-consistent, they give the general properties of diffuse equilibria for cases in which the applied helical fields contain only small $\ell$ components. A comparison with experimental results and self-consistent, three-dimensional numerical calculations 9,15 confirms the averaged results obtalined here. Experimentally, the design values for the applied helical fields for a diffuse plasma must be much larger than those required for a sharp-boundary plasma.

The model described here may be easily extended to describe long wavelength, $m=1$ Instabilities as pointed out by Bartsch and Miller.4,18 These unstable modes are drivan by the body force experienced by a displaced

TABLE IV

FIELD PRODUCT FOR DIFFUSE $\ell=1,2$ EQUILIBRIA

$\underline{B(0)}$

0.01

0.1

0.3

0.5

0.7

0.9

0.99
Rigid Rotor

1.003

1.026

1.082

1.144

1.208

1.249

1.206
Gaussian

1.004

1.025

1.081

1.145

1.220

1.302

1.331 
plasma column in the applied helical fielus. Since the simple model described above gives the body force experienced by a diffuse plasma in arbitrary helical. flelds, the ciestabilizing force may be easily included. Hence, much of the Important magnetohydrodynamic behavior of the high-beta stellarator may be described by the volume-averaged results obtained here.

\section{REFERENCES}

1. W. R. Fi: -. C. Jahoda, R. Kristal, W. E. Quinn, F. L. Ribe, G. i. E. Syer, and R. E. Slemon, "Plasma Equllibrium and Stability in the Scylla : Joroldal sector Experiments," Nucl. Fusion 14, 841 (1974).

2. E. L. Cantre11, W. R. Ellis, H. W. Harris, F. C. Jahoda, R. Kristal, M. D. Machalek, J. R. McConnell, W. E. Quinn, R. L. Ribe, G. A. Sawyer, F. T. Selbel, and R. E. Siemon, "Plasma Experiments in the Scyllac Toroldal Theta Pinch," in Plasma Physics and Controlled Nuclear Fusion Research (International Atomic Energy Agency, Vienna, 1975), Vol. 3 , p. 13.

3. R. R. Bartsch, C. J. Buchenauer, E. L. Cantre11, J. N. Dorning, B. L. Freeman, K. B. Freese, R. F. Gribble, I. Henins, A. R. Jacobson, F. C. Jahoda, T. R. Jarboe, R. Kristal, G. Miller, W. E. Quinn, F. L. Ribe, A. R. Sherwood, R. E. Siemon, and K. S. Thomas, "Plasma Experiwents on Staged Theta Pinch, Implosion Heating Experiment and Scyllac Feedback-Sector Experiment," In Plasma Physics and Controlled Nuclear Fusion Research (International Atomle Energy Agency, Vienna, 1977), Vo1. 2, p. 193. Atomtc Energy Agency, Vienna (1976).

4. R. R. Bartsch, E. L. Cantrell, R. F. Gribble, K. A. Klare, K. J. Kutac, G. Miller, and W. E. Quinn, "Feedback Stabilization of an $\ell=0,1,2$ High-Beta Stellarator," Nucl. Fusion 18, 1027 (1978).

5. W. Braun D. Correa, W. Engelhardt, E. Fünfer, J. Gruber, F. Herrnegger, M. Kaufmann, W. Köppendörfer, D. Lartz, W. Lotz, M. Münich, J. Neuhauser, W. Schnelder, G. Schramm, U. Selöel, and J. Sommer, "Garching Toroldal High-Beta Stellarator Experiments," in Plasma Physics and Controlled Nuclear Fusion Research (International Atomic Energy Agency, Vienna (1975), Vol. 3, p. 25.

6. E. Funfer, M. Kaufmann, W. Lotz, J. Neuhauser, G. Schramm, and U. Se1del, "High-Beta Stellarator Experiments on ISAR TI," Nucl. Fusion 15, 133 (1975).

7. F. Herrnegger M. Kaufmann, D. Lortz, J. Neuhauser, J. Nührenberg, W. Schneider, and G. Schramm, "Experimental and Theoretical Study of the FLR Stabilization in the High-Beta Stellarator-Equilibrium and Stability of MHD-Configurations with Zero Rotational Transform," In Plasma Physics and Controlled Nuclezr Fusion Research (International Atomlc Energy Agency, Vienna, 1977), Vol. 2, p. 183. 
8. 0. Betancourt and P. Garabedian, "Equilibrium and Stablity Code for a Diffuse Plasma," Proc. Nat. Acad. Sc1. USA 73, 984 (1976).

9. D. C. Barnes and J. U. Brackb111, "Computation of Magnetohydrodynamic Flow In a Magnetically Confined Plasma," Nucl. Sc1. and Engr. 64,18 (1977).

10. A. A. Blank, F. Grad, and H. Weltzner, In Proc. Conf. Plasma Physics and Controlled Nuclear Fusion Research 2, 607, International Atomic Energy Agency, Vienna (1969).

11. F. L. Ribe and M. N. Rosenbluth, "Feedback Stabilization of a High-B, Sharp-Boundaried Plasma Column with Helical Fields," Phys. Fluids 13 , 2572 (1970).

12. R. C. Davidson and J. P. Freldberg, "Review of Toroidal $\theta$-Pinch Theory," in Pulsed High Beta Plasmas, Edited by D. E. Evans, (Pergamon Press, Oxford, 1976): p 13 .

13. H. Weltzner, Private Communcation.

14. D. C. Barnes, J. U. Brackb111, R. Y. Dagazlan, J. P. Freldberg, W. Schneider, 0. Betancourt, "Analytic and Numerical Studies of Scyllac Equilibria," in Plasmá Physics and Controlled Nuclear Fuston Research, (International Atomic Energy Agency, Vienna, 1977), Vol. 2, p. 203.

15. D. C. Barnes, J. U. Brackb111, and W. Schnelder, "A Numerical Study of High-Beta Stellarator Equilibria," submitted to Phys. Fluids.

16. G. Berge and J. P. Freidberg, "Stabillty of a Diffuse, High- $\beta$, $\ell=1$ System," Phys. Flu1ds 18, 1362 (1975).

17. P. C. T. van der Laan, "Drift of a Plasma in a Curved ragnetic Field," J. Nucl. Energy Part C 6, 559 (1964).

18. G. Miller, "Long Wavelength $m=1$ Magnetohydrodynamics of a Theta Pinch," Phys. Fluids 20, 928 (1977). 